\title{
Raw Dataset for Final Analysis
}

National Cancer Institute

\section{Source}

National Cancer Institute. Raw Dataset for Final Analysis. NCI Thesaurus. Code C115494.

The collection of unprocessed data records used for a final analysis of results for a clinical trial. 